\title{
Effect of Aqueous Extract of Naja naja Sp. Shedded Skin in Pregnant Female Rats and its Role on the Growth and Develop- ment in the Pups
}

\begin{abstract}
Keywords: Pregnancy; Prenatal toxicity; Snake shed skin; SSS
\section{Abstract}

Snake shed skins are widely used in the folk and traditional medicine of various cultures since ancient time. In ancient Chinese and Levantine medicinal system snake shed skin is used in the treatment of several diseases viz. glaucoma, hernia, psoriasis etc. Indian traditional and folk medicinal system make use of the ash of snake shed skin for inducing labour in pregnant women, which lacks scientific validation. Mukherjee et al., 2013 reported that $N$. naja shedded skin caused temporary cessation of the estrous cycle. However no scientific evidence is present regarding its role on pregnant animals. Thus, the present study was commenced for scientifically validating the role of Naja naja shed skin aqueous extract on the pregnant rats.

In the present study, sss altered the urinary volume, calcium, magnesium levels, as well as altered the urinary creatinine output. SSS also altered serum 17- $\beta$ estradiol level, progesterone and C-reactive protein level. In a dose-dependent manner, SSS exposure towards pregnant mother retarded the growth and development in the pups. At the highest dose SSS lead to fetal resorption. The present study for the first reports the prenatal toxicity and teratogenicity of SSS.
\end{abstract}

\section{Abbreviations}

b.w: Body Weight; P4: Progesterone; s.c: subcutaneous; SSS: Naja naja Shedded Skin aqueous extract; SSS(1): Naja naja Shedded Skin aqueous extract 1 mg.kg-1 b.w.; SSS(10): Naja naja Shedded Skin aqueous extract $10 \mathrm{mg} . \mathrm{kg}^{-1}$ b.w.; SSS(5): Naja naja Shedded Skin aqueous extract $5 \mathrm{mg} \cdot \mathrm{kg}^{-1}$ b.w.

\section{Introduction}

Snake and its body parts, are studied extensively in the folk and traditional medicine of various cultures, since primeval time for therapeutic benefits [1-3]. Evidences based studies in Chinese and Levantine medicinal system revealed the use of snake shed skin for treatment of several diseases. In the Indian traditional medicine, snake shed skin is used for labour induction in pregnant women [4]. Though largely used by traditional naturopaths for treating various female reproductive disorders, there is no scientific evidence regarding the bioactivity of the snake shed skin on female reproductive system.

Reported for the first time the role of aqueous Naja naja shed skin extract on the estrous cycle of female mice [4]. The Naja naja shedded skin aqueous extract caused cessation of the estrous cycle for 10 days by altering hormone ( $\mathrm{LH}, \mathrm{FSH}, 17-\beta$ estradiol, progesterone) and cytokine (IL-1 $\beta$, TNF- $\alpha$, IL-12) profiles. Although the role of the aqueous extract on the estrous cycle of mice are quite extensively

\section{Journal of}

Toxins

\author{
Mukherjee S $^{1}$, Gomes $\mathrm{A}^{2}$ and Das Gupta SC ${ }^{1 *}$ \\ ${ }^{\prime}$ Department of Zoology Maulana Azad College, Rafi Ahmed Kidwai \\ Road, India \\ ${ }^{2}$ Laboratory of Toxinology \& Experimental Pharmacodynamics, \\ Deptartment of Physiology, Calcutta University, India
}

*Address for Correspondence

Das Gupta SC, Department of Zoology Maulana Azad College, 8, Rafi Ahmed Kidwai Road, Kolkata 700013, India, Tel: 91-33-2226 0995; Fax-9133--2226-0995; Mobile: + 91-9830471981; E-mail: subirdgupta@gmail.com

Submission: 27 May, 2019

Accepted: 31 July, 2019

Published: 03 August, 2019

Copyright: $\odot 2019$ Mukherjee S, et al. This is an open access article distributed under the Creative Commons Attribution License, which permits unrestricted use, distribution, and reproduction in any medium, provided the original work is properly cited.

studied, but its role on the pregnant female rats remains vague. Therefore, the present study is embarked to evaluate the role of Naja naja shed skin aqueous extract on pregnant female rats, and subsequently its effect on pups.

\section{Materials and Methods}

\section{Chemicals and reagents}

The following kits were use for the present study: Calcium, creatinine, magnesium were purchase from Ecoline Merck (India). $17-\beta$ Estradiol $\left(\mathrm{E}_{2}\right)$, progesterone $\left(\mathrm{P}_{4}\right)$, C-Reactive Protein (CRP) Elisa kits were purchase from Cusabio (China).

Collection of N. naja shedded skin- Fresh Naja naja shedded skins of both sexes was collected from North 24-Parganas, West Bengal, India through field collection as per the permission granted by the Ministry of Forests \& Wild Life, Govt. of West Bengal, India (2105/ WL/4R-l (PI-IX)) and identified by the Zoological Survey of India. The shedded skins were store in desiccator at room temperature and later subjected to aqueous extraction.

\section{Animals and experimental design}

Sexually matured male and female Wistar rats were purchase from the enlisted supplier of Maulana Azad College, Kolkata. During breeding and until gestation day, rats were housed in polypropylene cages at $22 \pm 3{ }^{\circ} \mathrm{C}$; relative air humidity 45 to $55 \%$ with $12.00 \mathrm{~h}$ light \& dark cycle and provided with standard diet and tap water ad libitum. The rats were acclimatized for one week in the laboratory conditions, before being used in the experiment. Virgin female rats (approximately120-130 g) were bred overnight with untreated adult males of the same strain (one male: two females) [5]. The day on which sperm were visualized in the vaginal smear was designated 0 day pregnant. Sperm positive females were randomly assigned to test groups according to their day 0 of gestation. All experimental protocols described in the present study are approved by institutional animal ethics committee (CPCSEA), Govt. of India (Institutional Sanction No: 25/ 250/ 2012 - AWD).

Preparation of aqueous extract of Naja naja Shed Skin (SSS) and protein estimation 

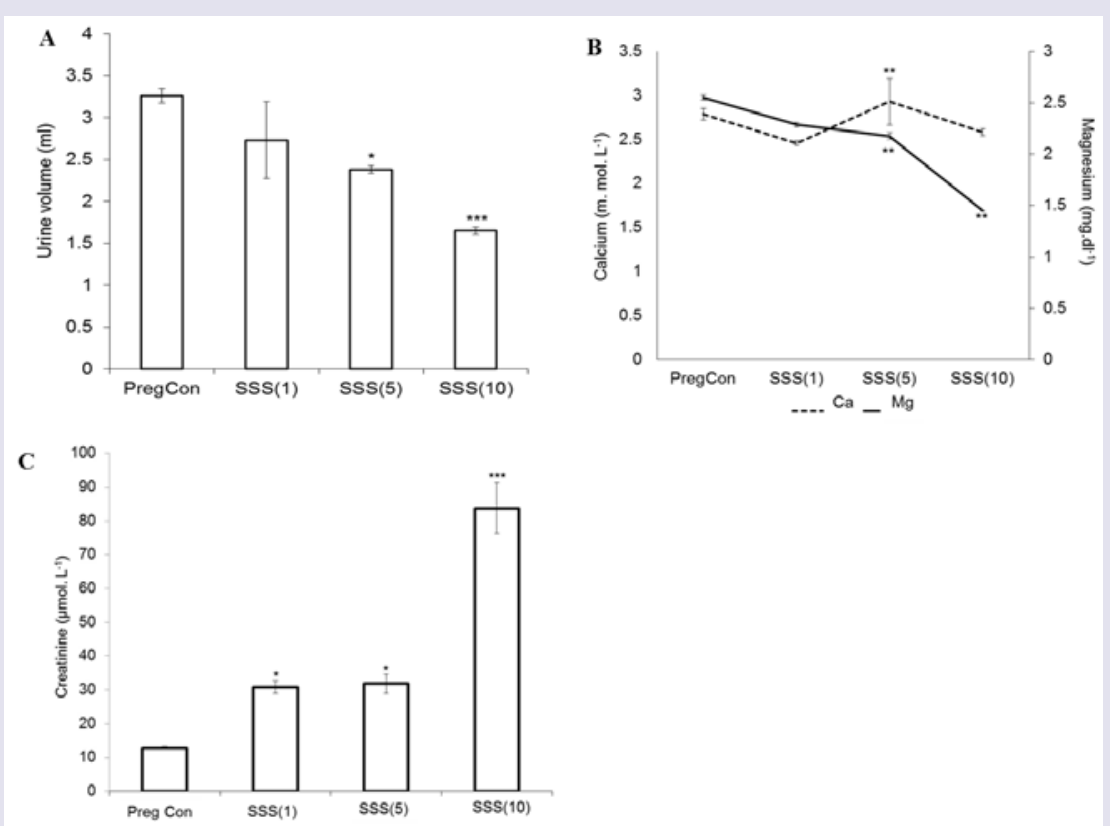

Figure 1: Effect of SSS on urinary biochemical markers.

0 day pregnant rats were treated with SSS(1) (1 mg.kg-1 body weight, X 10 days, s.c), SSS(5) (5 mg.kg-1 body weight, X 10 days, s.c) and SSS(10) (10 mg.kg-1 body weight, $X 10$ days, S.C). (A) SSS(1) did not produce significant change in the urinary volumes on day 20 of gestation in pregnant rat as compared with pregnant control rat. SSS(5) and SSS(10) produced significant decrease in the urinary volumes on day 20 of gestation in pregnant rat as compared with pregnant control rat.

(B) SSS(1) did not produce significant change in the urinary calcium and magnesium level on day 20 of gestation as compared with control pregnant rat. SSS(5) produced significant increase in urinary calcium on day 20 of gestation but SSS(10) did not produced significant change in urinary calcium as compared with control pregnant rat. SSS(5) and SSS(10) produced significant decrease in urinary magnesium on day 20 of gestation as compared with control pregnant.

(C) SSS(1) SSS(5) and SSS(10) produced significant increase in the urinary creatinine on day 20 of gestation in pregnant rat as compared with control pregnant rat Values were represented as mean \pm SEM $(n=4) .{ }^{*} P<0.05,{ }^{* * *} P<0.001,{ }^{* * *} P<0.001$ (Significant).

Freshly powdered Naja naja shedded skin was soaked in physiological mammalian saline overnight at $4^{\circ} \mathrm{C}$. It was strained, and centrifuged at $5000 \mathrm{rpm}$ at $4^{\circ} \mathrm{C}$ for $20 \mathrm{~min}$. Supernatant was collected and expressed in terms of protein content [6]. The supernatant was used for the experimental study.

Treatment regime- For study with aqueous extract of snake shed skin extract (SSS) and control animals were randomized into 4 groups $(\mathrm{n}=4)$ : Control pregnant, SSS(1) $\left(1 \mathrm{mg} \cdot \mathrm{kg}^{-1}\right.$ b.w. $)$, SSS(5) $\left(5 \mathrm{mg} \cdot \mathrm{kg}^{-1}\right.$ b.w.) and SSS(10) (10 mg.kg-1 b.w.). SSS(1), SSS(5) and SSS(10) were administered sub cutaneously (s.c.) from day 0 to day 9 of gestation (total of 10 exposure).

Effect of SSS on urinary biochemical markers- Urine was collected on day 20 (final trimester), urinary volume was measured, calcium, magnesium, creatinine were assayed.

Effect of SSS on serum biochemical markers- On day 21 blood was collected from retro-orbital plexus of the rats, and the serum was separated. From the serum; hormones $\left(\mathrm{E}_{2}, \mathrm{P}_{4}\right)$, and inflammatory marker (CRP) were assayed by Elisa (Biotek, USA; Model No. EL $\times 800$ MS) according to the manufacturers protocols. The concentration of these hormones and CRP were calculated from their respective standard curves.

Biomorphometry of pups- Gestational period of the pregnant female rats were monitored after prenatal exposure of SSS $(1,5 \& 10$ mg.kg-1 body weight X 10 exposure, s.c.) in pregnant rat. Following gestational period and the birth of pups, the bio-morphometric features (body weight, head length, head diameter, neck width and tail length) were monitored and measured every 5 days interval (using digital calliper Mitutoyo, Japan), until the weaning period (21 days) is completed.

Statistical analysis- Statistical analysis was done by Graph Pad InStat software (La Jolla, CA, USA). Data were expressed as mean \pm SEM $(n=4)$ unless otherwise mentioned. The differences between the treated and untreated control group were analyzed by one-way ANOVA and post-test was done using Tukey's multiple comparison tests to determine the significant levels. ${ }^{*} \mathrm{p}<0.05$, ${ }^{* *} \mathrm{p}<0.01,{ }^{* *} \mathrm{p}<0.001$ were considered significant.

\section{Results}

The protein content of Naja naja aqueous extract (SSS) was found to be $6 \pm 1 \mathrm{mg} \cdot \mathrm{ml}^{-1}$.

\section{Effect of SSS on urinary biochemical markers}

SSS(1) treatment did not produced significant change in the urinary volume on day 20 of gestation as compared with pregnant control rat. SSS(5) and SSS(10) treatment produced significant decrease in the urinary volume on day 20 of gestation as compared with pregnant control rat (Figure 1a). SSS(1) treatment did not produce significant change in urinary calcium level on day 20 of gestation as compared with pregnant control rat. SSS(5) treatment produced significant increase in the urinary calcium level on day 20 of gestation as compared with pregnant control rat. SSS(10) did 
Citation: Mukherjee S, Gomes A, Das Gupta SC. Effect of Aqueous Extract of Naja naja Sp. Shedded Skin in Pregnant Female Rats and its Role on the Growth and Development in the Pups. J Toxins. 2019;6(1): 5

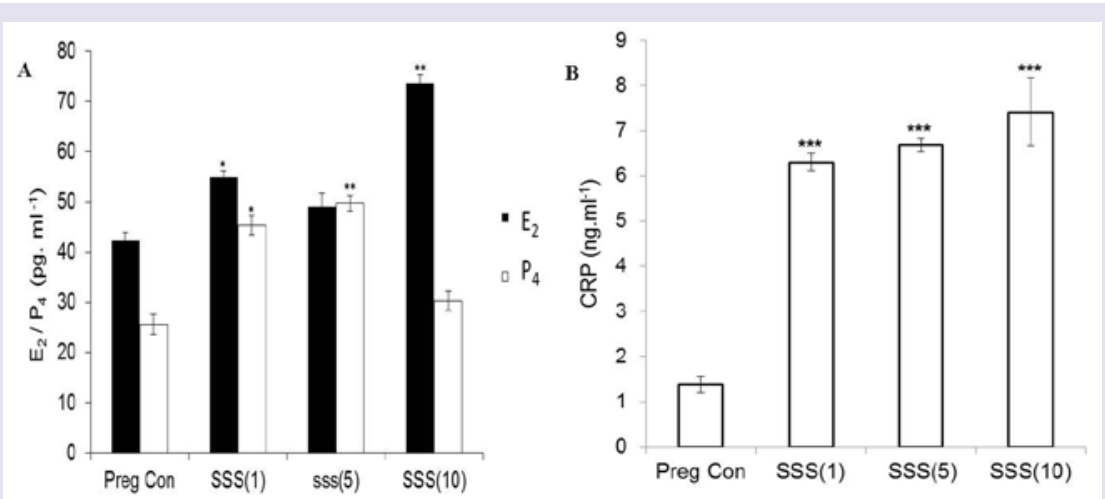

Figure 2: Effect of SSS on serum biochemical markers.

0 day pregnant rats were treated with SSS(1) (1 mg.kg- 1 body weight, $X 10$ days, s.c), SSS(5) (5 mg.kg-1 body weight, X 10 days, s.c) and SSS(10) (10 mg.kg-1 body weight, $X 10$ days, S.c). (A) SSS(1) produced significant increase in serum E2 and P4 on day 21 of gestation as compared with control pregnant rat. SSS(5) did not produce significant change in serum E2 and produced significant increase in serum P4 on day 21 of gestation as compared with pregnant control rat. SSS(10) produced significant increase in serum E2 level and did not produced significant change in serum P4 on day 21 of gestation as compared with control pregnant rat.

(B) SSS(1), SSS(5) and SSS(10) produced significant increase in serum CRP on day 21 of gestation as compared with control pregnant rat. Values were represented as mean \pm SEM $(n=4) .{ }^{*} P<0.05,{ }^{* * *} P<0.001,{ }^{* * *} P<0.001$ (Significant)
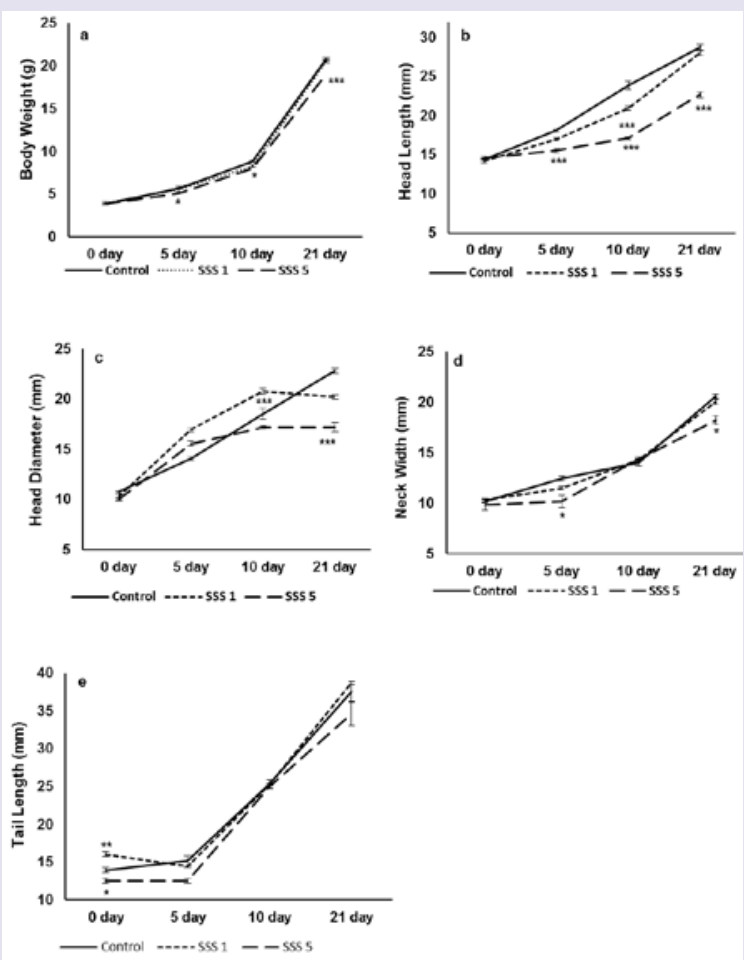

Figure 3: Effect of SSS on the biomorphometric parameters of pups.

0 day pregnant rats were treated with SSS(1) (1 mg.kg-1 body weight, X 10 days, s.c), SSS(5) (5 mg.kg-1 body weight, X 10 days, s.c) and SSS(10) (10 mg.kg-1 body weight, $X 10$ days, s.c) . a: body weight in $\mathrm{g}$; b: head length in $\mathrm{mm}$, c: head diameter in $\mathrm{mm}, \mathrm{c}$ : neck width in $\mathrm{mm}$, d: tail length in $\mathrm{mm}$.

Values were represented as mean \pm SEM $(n=7) .{ }^{*} P<0.05,{ }^{* * *} P<0.001,{ }^{* * *} P<0.001$ (Significant).

not produce significant change in the urinary calcium level on day 20 of gestation as compared with pregnant control rat (Figure 1b). SSS(1) treatment did not produce significant change in the urinary magnesium level on day 20 of gestation as compared with pregnant control rat. SSS(5) and SSS(10) treatment produced significant decrease in urinary magnesium level on day 20 of gestation as compared with pregnant control rat (Figure 1b). SSS(1), SSS(5) and SSS(10) treatment produced significant increase in the urinary creatinine level on day 20 of gestation as compared with pregnant control rat (Figure 1c).

Effect of SSS on serum biochemical markers- SSS (1) treatment produced significant increase in the serum E2 level on day 21 of gestation as compared with pregnant control rat. SSS(5) treatment did not produce significant change in serum E2 level on day 21 of 

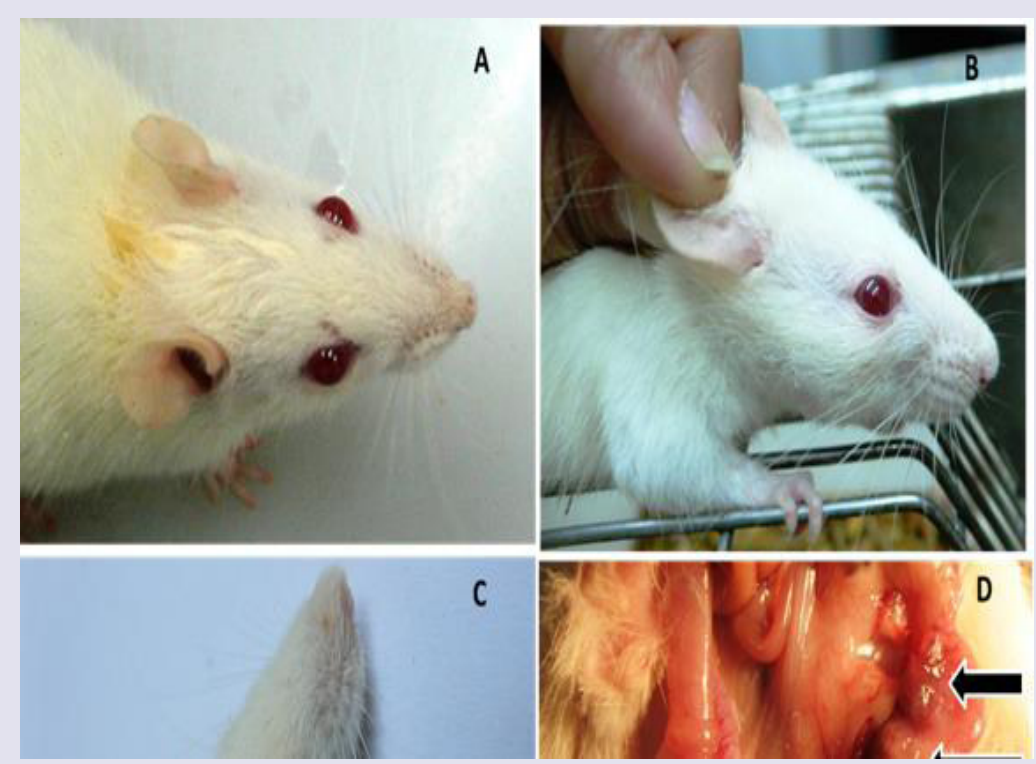

Figure 4: Effect of SSS treatment on pregnant femats and its consequence on the pups.

0 day pregnant rats were treated with SSS(1) (1 mg.kg-1 body weight, $X 10$ days, s.c), SSS(5) (5 mg.kg-1 body weight, X 10 days, s.c) and SSS(10) (10 mg.kg-1 body weight, $X 10$ days, S.C). (A) Control Pups: showed normal development including no impairment in eye (B) SSS(1) treated pups: showed normal development including no impairment in eye, (C) SSS(5) treated pups showed: developmental toxicity as is evident improper development of the eye. (D) a. SSS(10) caused fetal resorption. SSS(10) treated pregnant rats showed sites of implantation sites even on 30 post gestation days. Data shown here were from one of the four repeated experiment showing similar results.

gestation as compared with pregnant control. SSS(10) treatment produced significant increase in serum E2 level on day 21 of gestation as compared with pregnant control rat (Figure 2a). SSS(1) and SSS(5) treatment produced significant increase in serum P4 level on day 21 of gestation as compared with pregnant control rat. SSS(10) treatment did not produce significant change serum P4 level on day 21 of gestation as compared with pregnant control (Figure 2a). SSS(1), SSS(5) and SSS(10)treatment produced significant increase in of serum C-reactive protein (CRP) level on day 21 of gestation as compared with pregnant control rat (Figure 2b).

Effect of SSS on biomorphometry and physiological parameters of the pups- SSS(1) treatment did not produce significant change in body weight, head diameter, neck width and tail length of the pups on day 0 up to day 21 as compared with control pups. SSS(1) treatment produced significant decrease in the head length of the pups on day 5 , on day 10 which returned to control level as compared with control pups. (Figure $3 \mathrm{a}$ and $3 \mathrm{~b}$ ).

SSS(5) treatment caused growth impairment in the pups. It led to malformation in the head region. The eyes were not well developed (Figure 4c), but no malformation was observed in the four digits in the front and hind limb. SSS(5) treatment did not produce significant change in body weight from day 0 to day 15, but it significantly decreased on day 21 as compared with control pups (Figure 3a). SSS(5) treatment produced significant decrease in the head length on day 0 up to day 21 as compared with control pups (Figure $3 b$ ). SSS(5) treatment did not produce significant change in head diameter observed on day 0 to day 15 , but significantly decreased on day 21 as compared with control pups (Figure 3c). SSS(5) treatment did not produce significant change in neck width from day 0 to day 15 , but significantly decreased on day 21 as compared with control pups
(Figure 3d). SSS(5) treatment did not produced significant change in tail length on day 0 to day 21 as compared with control pups (Figure 3e).

SSS(10) treatment caused fetal resorption, and absence of birth of new born pups as was evident from the sites of implantation upon examination of the uterus. (Figure $4 \mathrm{~d}$ ).

\section{Discussion}

The present study was designed to evaluate the role of Naja naja shed skin aqueous extract on pregnant female rats. In the present study, SSS decreased urinary volume in a dose dependent manner. SSS(5) increased urinary calcium output, SSS(5) and SSS(10) decreased urinary magnesium and increased urinary creatinine output. During normal pregnancy, urinary volume is increased at second and third trimester which is mediated by angiotensin-(1-7) [7]. This is associated with increased water intake, decreased plasma vasopressin and down regulation of kidney Aquaporin 1 (AQP1) [8]. Mineral metabolism is regulated by the demand of the fetus and placenta, which together pulled calcium and other minerals from the maternal circulation for the growth, development and various enzymatic reactions across feto-placental barriers [9,10]. During pregnancy creatinine acted as one of the most important indices for muscle mass loss [11]. From the above result it can be concluded that SSS altered normal mineral status as well as water balance indicating it altered normal pregnancy condition (Figure 1a-1d).

Pregnancy remained under the influence of two major steroidal hormones; estrogen and progesterone. Progesterone was mainly required for maintaining the quiescent endometrium preventing preterm delivery. Just prior to labour there was increase in the level of estrogen 100-1000 folds and in turn shifted the uterus from quiescent 
Citation: Mukherjee S, Gomes A, Das Gupta SC. Effect of Aqueous Extract of Naja naja Sp. Shedded Skin in Pregnant Female Rats and its Role on the Growth and Development in the Pups. J Toxins. 2019;6(1): 5

to rhythmically contractile state. In the present study SSS(1) and SSS(5) treatment produced significant rise in $17-\beta$ estradiol and decreased progesterone as compared with pregnant control rat [12]. SSS(10) treatment showed decrease in estradiol and an increase in progesterone due to fetal resorption (Figure 2a). Serum C-reactive protein is a major acute phase inflammatory protein. During normal pregnancy, an increase in the level C-reactive protein in labour indicated muscle damage and tearing associated with parturition, but a very high and elevated level was associated with preterm delivery, fetal growth restriction and preeclamsia $[13,14]$. SSS(1) and SSS(5) treatment produced dose dependent increase in the C-Reactive Protein (CRP) at term as compared with pregnant control supported that SSS had activated the inflammatory pathway associated with parturition. SSS(10) treatment caused a further increase in CRP due to fetal resorption where membrane damage was more pronounced.

In the pups SSS(1) did not produce significant change in body weight, head diameter, neck width and tail length but decreased head length; and SSS(5) decreased body weight, head diameter, neck width and head length of the pups. In the SSS(5) treated pups, the eyes were not developed indicating that SSS had passed feto-placental barrier, caused growth restriction and hampered the developmental process. SSS(10) due to feto-placental passage caused fetal resorption by recruiting the inflammatory markers $[15,16]$.

The above study reflected that SSS is associated with reproductive toxicity in pregnant rat associated with developmental toxicity and teratogenic effect.

\section{References}

1. Costa-Neto EM (2005) Animal-based medicines: biological prospection and the sustainable use of zootherapeutic resources. An Acad Bras Cienc 77 33-43.

2. Soewu DA (2008) Wild animals in ethnozoological practices among the Yorubas of southwestern Nigeria and the implications for biodiversity conservation. Afr J Agric Res 6: 421-427.
3. Lev E (2003) Traditional healing with animals (zootherapy): medieval to present-day Levantine practice. J Ethnopharmacol 85: 107-118.

4. Mukherjee S, Dasgupta SC, Gomes A (2013) Effect of Naja naja Laurenti shed skin extract on estrous cycle, hormone - cytokine profiles, histopathology of ovary and uterus of Swiss albino mice. Indian J Exp Biol 51: 235-240.

5. Hellwig J, Liberacki AB (1997) Evaluation of the pre-, peri-, and postnatal toxicity of monoethanolamine in rats following repeated oral administration during organogenesis. Fundam Appl Toxicol 40: 158-162.

6. Lowry OH, Rosebrough NJ, Farr AL, Randall RJ (1951) Protein measurement with the Folin phenol reagent. J Biol Chem 193: 265-275.

7. Lubrano Rosales A, Chesa Ponce N (1999) Study of urinary outflow in pregnancy. Actas Urol Esp 23: 763-768.

8. Joyner J, Neves LA, Stovall K, Ferrario CM, Brosnihan KB (2008) Angiotensin-(1-7) serves as an aquaretic by increasing water intake and diuresis in association with downregulation of aquaporin-1 during pregnancy in rats. Am J Physiol Regul Integr Comp Physiol 294: R1073-R1080.

9. Kovacs CS, Gel-H F (2006) Calcium and bone disorders during pregnancy and lactation. Endocrinol Metab Clin North Am 35: 21-51.

10. Kovacs CS (2013) Bone metabolism in the fetus and neonate. Pediatr Nephrol 29: 793-803.

11. Phillips DI (1995) Relation of fetal growth to adult muscle mass and glucose tolerance. Diabet Med 12: 686-690.

12. Hendrick V, Altshuler LL, Suri R (1998) Hormonal changes in the postpartum and implications for postpartum depression. Psychosomatics 39: 93-101.

13. Ernst GD, de Jonge LL, Hofman A, Lindemans J, Russcher H, et al. (2011) $\mathrm{C}$-reactive protein levels in early pregnancy, fetal growth patterns, and the risk for neonatal complications: the Generation R Study. Am J Obstet Gynecol 205: 132.e1-e12.

14. van den Hooven $\mathrm{EH}$, de Kluizenaar $\mathrm{Y}$, Pierik FH, Hofman $\mathrm{A}$, van Ratingen SW, et al. (2012) Chronic air pollution exposure during pregnancy and maternal and fetal C-reactive protein levels: the Generation R Study. Environ Health Perspect 120: 746-751.

15. Konopka CK, Morais EN, Naidon D, Pereira AM, Rubin MA, et al. (2013) Maternal serum progesterone, estradiol and estriol levels in successful dinoprostone-induced labor. Braz J Med Biol Res 46: 91-97.

16. Kovacs CS, Kronenberg HM (1997) Maternal-fetal calcium and bone metabolism during pregnancy, puerperium, and lactation. Endocr Rev 18: 832-872.

Acknowledgement

We thankfully acknowledge University Grant Commission, New Delhi, India for partial financial assistance (Ref No: F 38-130 (SR), dated 19.12.2009 and we are thankful to the Dept. of Forests \& Wild Life, Govt. of West Bengal, India (2105/WL/4R-1(PI- IX)) for kind permission of collection of the shed snake skin. 